\title{
La neurociencia para la inclusión en contextos pluriculturales
}

\author{
Neuroscience for inclusion in multicultural contexts \\ Neurowissenschaften für die Einbeziehung in multikulturelle Kontexte \\ Les neurosciences pour l'inclusion dans les contextes multiculturels \\ Neurociência para inclusão em contextos multiculturais \\ Neuroscienze per l'inclusione in contesti multiculturali \\ Нейронаука для включения в многокультурный контекст \\ 多文化の文脈におけるインクルージョンのための神経科学 \\ 神经科学促进多元文化背景下的包容。
}

\author{
Cristina Pinto Díaz \\ Universidad de Jaén (España) \\ cpd00008@red.ujaen.es
}

Fecha recepción: 21/10/2020

Páginas 1-8

Fecha aceptación: 29/12/2020

\section{Resumen.}

Es importante la neurociencia para la inclusión en contextos educativos pluriculturales. El objetivo de esta investigación es comparar las opiniones del alumnado de Grado de Educación Primaria y Máster de Profesorado en relación a la presencia de la neurociencia en el aula pluricultural para fomentar la inclusión. Este estudio parte de la siguiente hipótesis: "La neurociencia para una inclusión educativa favorece contextos educativos pluriculturales". Para tratar de dar respuesta a este objetivo y a la hipótesis de partida se ha desarrollado una investigación descriptiva, explicativa y correlacional basada en una metodología cuantitativa. Para la investigación se ha elaborado una escala Likert, para ello, en primer lugar, se ha construido una tabla de operacionalización con 6 dimensiones de 6 ítems en cada dimensión y con un Alpha de Cronbach de $\alpha=.836$ en la escala de los estudiantes de Grado y $a=.795$ en la escala de los estudiantes de Máster. El instrumento se aplicó a una muestra de 296 sujetos estudiantes de Grado y 295 sujetos de Máster de una universidad andaluza. Se concluyó que los estudiantes de Grado y los de Máster están concienciados sobre la importancia de la neurociencia para la inclusión en contextos pluriculturales.

Palabras clave: neurociencia; inclusión; pluriculturalidad; contextos educativos; contextos pluriculturales; contextos inclusivos

\section{Abstract.}

Neuroscience is important for inclusion in multicultural educational contexts. The objective of the research is to compare the opinions of Primary Education Degree's students and students of Teachers Master's regarding the presence of neuroscience in the multicultural classroom to encourage inclusion. This study is based on the following hypothesis: "neuroscience for educational inclusion favors multicultural 
educational contexts". In order to try to respond to this objective and the starting hypothesis, it has been developed a descriptive, explanatory and correlal research based on a quantitative methodology. For the research, a Likert scale has been developed, for this purpose, first, an operationalization table has been built with 6 dimensions of 6 items in each dimension and with a Cronbach Alpha of $a=.836$ on the scale of the Degree's students and $a=.795$ on the scale of the Master's students. The instrument was applied to a sample of 296 Degree's subjects and 295 Master's subjects from an Andalusian university. It was concluded that Degree's students and Master's students are aware of the importance of neuroscience for inclusion in multicultural contexts.

Keywords: neuroscience; inclusion; multiculturalism; educational contexts; multicultural contexts; inclusive contexts

\section{1.-Introducción.}

Las tendencias científicas en educación están formadas por disciplinas científicas y académicas que observan la actividad cerebral y los comportamientos de la persona (Gago y Elgier, 2018). Estas tendencias científicas incluyen neuroeducación la cual aúna la neurología y la educación según la plasticidad cerebral (Espino-Díaz, Álvarez-Castillo, González-González, Hernández-Lloret y Fermández-Caminero, 2020; Arraya-Pizarro y Espinoza, 2020). Además, también engloba la neurodidáctica que defiende la educación teniendo en cuenta la pluralidad y necesidades del alumnado. También emerge el término conocido como neurodiversidad para referirse a la diversidad cerebral de la persona (Rahman y Woolard, 2019).

Debido al sistema educativo globalizado, es necesario que los docentes se formen en neurociencia para mejorar el proceso de enseñanza-aprendizaje y asegurar una educación inclusiva, dejando a un lado la discapacidad para centrarse en la adaptación del material (Espino-Díaz et al., 2020; Arraya-Pizarro y Espinoza, 2020; Rahman y Woolard, 2019).

Actualmente, la educación inclusiva preocupa a toda la comunidad educativa (Muntaner, 2019). La escuela tiene el deber de crear una cultura inclusiva, de igualdad y respeto a todos los niños y niñas (Vidal, 2019). Además, la escuela debe cumplir con el derecho a una educación equitativa y universal, con una cultura, políticas y prácticas inclusivas, igualitarias y respetuosas, prestando más cuidado al alumnado excluido o en riesgo de exclusión social para que en el futuro sean ciudadanos activos y participativos, críticos y solidarios (Florian y Camedda, 2020). Por ende, todo el alumnado tiene derecho a recibir una educación inclusiva y de calidad, independientemente de sus capacidades y aptitudes (Ainscow, 2017).

Además, el profesorado debe estar formado en inclusión para tener una actitud positiva hacia la inclusión (Medina-García, Doña-Toledo y Higueras-Rodríguez, 2020).

En lo referente a la cultura podemos diferenciar varios términos similares entre sí, pero con pequeños matices que los diferencian. La cultura se puede definir como las diferentes manifestaciones emocionales, físicas, intelectuales, etc. de una sociedad (UNESCO, 1982). La cultura engloba términos como pluriculturalidad que se puede definir como las culturas de un mismo espacio geográfico sin intercambios culturales 
(Bernabé, 2012). Otro término es la multiculturalidad la cual previene de conflitos y promueve la convivencia pacífica de distintas culturas (De Barros y Hernández, 2018; Luz, 2018). La interculturalidad valora positivamente la diversiad (Aguado, 2016; De Barros y Hernández, 2018). Desde la educación la interculturalidad reflexiona obre la diversidad y la riqueza que esta proporciona en contextos educativos (Dietz, 2018; Fylkesnes, 2018; Leiva, 2019). La educación intercultural se basa en la equidad, la diversidad, la inclusión y la democracia. Fomenta la igualdad, la formación y la positividad de la diversidad cultural con un diálogo crítico para crear conciencia cultural crítica y justicia social para dar importancia al aprendizaje en contextos de la comunidad (Salgado-Orellana, Berrocal y Sánchez-Núñez, 2019). La intraculturalidad es la relación de una cultura consigo misma para el desarrollo de la identidad de la propia cultura (Jane, 2019). La transculturalidad es la unión entre las culturas para establecer vínculos y crear hechos culturales nuevos, con el objetivo de conseguir el respeto y promoción de valores universales (De Barros y Hernández, 2018).

En nuestros días es importante que el profesorado esté formado en neurociencia e inclusión para crear un sistema educativo equitativo y de calidad que respete la diferencia y la tome como positiva en aquellos contextos pluriculturales de las aulas actuales (Leiva, 2019).

\section{2.-Metodología.}

El problema que se nos plantea en esta investigación sería el siguiente: ¿La neurociencia favorece una inclusión educativa en los contextos educativos pluriculturales? El Objetivo general trata de comparar las opiniones del alumnado de Grado de Educación Primaria y Máster de Profesorado en relación a la presencia de la neurociencia en el aula pluricultural para fomentar la inclusión.

\section{1.-Diseño de la investigación.}

Esta investigación es de índole no experimental, exploratorio, descriptivo y correlacional y se ha efectuado mediante una metodología cuantitativa. Por ello, para la recogida de datos se ha aplicado una escala tipo Likert. Además, el software utilizado ha sido el paquete estadístico SPSS v.26.

\section{2.-Población y muestra.}

Se toma como población a 300 estudiantes del Grado de Educación Primaria y a 300 estudiantes del Máster de Profesorado, ambos de la Universidad de Jaén. Si tenemos en cuenta las ausencias, nuestra muestra será de 296 sujetos del Grado y 295 del Máster.

\section{3.-Instrumento para la recogida de datos.}

Para la recopilación de datos se confeccionó una matriz de operacionalización coordinando variables, ítems y unidades de medida (Mejía, 2005). Todo fue destinado para el diseño de una escala Likert de 36 ítems, agrupados en seis 
dimensiones: A (Tendencias Científicas), B (Inclusión Educativa), C (Pluriculturalidad), D (Contextos Educativos), E (Contextos Pluriculturales) y $F$ (Contextos Inclusivos).

Por ello, las variables las clasificaremos en:

-Las variables dependientes: Tendencias Científicas e Inclusión Educativa.

-Las variables independientes: Pluriculturalidad, Contextos Educativos, Contextos

Pluriculturales y Contextos Inclusivos.

\section{4.-Validez de contenido.}

En cuanto a la validez, primero, se procedió a una validez de contenido que se ejecutó por especialistas doctores de diversas universidades (Malla y Zabala, 1978) autorizados para esta evaluación, para los especialistas se calculó el Coeficiente de Conocimiento o Información ( $\mathrm{Kc}$ ) y el Coeficiente de Argumentación (Ka), y a continuación se calculó el valor del Coeficiente de Competencia $(K)$ para decidir qué expertos se tienen en cuenta para trabajar en esta investigación, se obtubieron quince especialistas con una $\mathrm{K}$ media de 0.9 , es decir, un nivel de competencia alto (Fernández, López y Márquez, 2008). Tras analizar los cuestionarios de validación se reajustaron algunas preguntas, sin afectar al fondo de la cuestión. También, se realizó una prueba piloto a un subgrupo de la muestra para revisar dificultades de comprensión, identificar las preguntas que generaban duda, etc., se utilizó la correspondiente lista de control (Iraossi, 2006). Los resultados de la prueba piloto fueron satisfactorios por lo que se tomó como validado el instrumento en su contenido.

\section{3.-Resultados.}

\section{1.-Fiabilidad.}

El coeficiente del Alfa de Cronbach muestra una aceptable-buena consistencia interna del conjunto de estas 36 variables ya que, por un lado, presenta un valor de a $=.836$ para los datos recogidos en la escala del Grado de Educación Primaria, que es un valor bueno. Por otro lado, los datos recogidos a través de la Likert del alumnado del Máster muestra u valor de $\alpha=.795$, que indica un valor aceptable-bueno (George y Mallery, 2003)

\section{2.-Bondad de ajuste.}

Según la prueba de Kolmogorov-Smirnov se debe "rechazar la Hipótesis nula", esto quiere decir que los datos no siguen una distribución normal. Por ello, se define la siguiente hipótesis alternativa: $\mathrm{H}_{1 .-}$ La neurociencia para una inclusión educativa favorece contextos educativos pluriculturales. 
Revista Internacional de Apoyo a la Inclusión, Logopedia, Sociedad y Multiculturalidad.

Volumen 7, Número 1, enero 2021, ISSN: 2387-0907. DOI: https://dx.doi.org/10.17561/riai.v7.n1.1

\section{3.-Análisis correlacional.}

Ya que en la prueba de Kolmogorov-Smirnov la distribución es anormal se utiliza la correlación de Spearman.

Analizando los ítems de la investigación, la correlación significativa (0.05) se establece entre las siguientes variables:

Fig. 1.-Correlaciones en la escala de Grado:
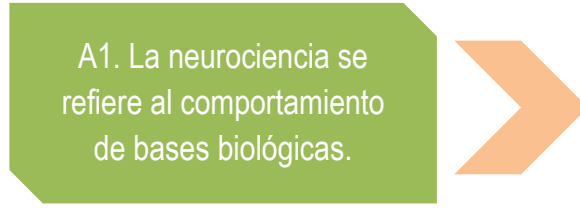

C14. La pluriculturalidad son diferentes culturas

que existen en el mundo.

F33. Los contextos

inclusivos deben

adaptarse al alumnado.

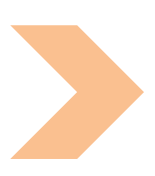

F34. En los contextos

inclusivos el aprendizaje se

produce desde la

colaboración y cooperación.

A3. La neurodidáctica

establece estrategias que el

cerebro utiliza para aprender.

C18. La transculturalidad es

la interacción de costumbres

de otras culturas para

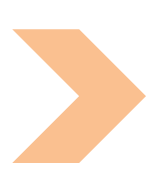

Correlaciones de la escala de Máster:

\section{B7. La inclusión
educativa pretende
responder a las
necesidades del \\ B7. La inclusión
educativa pretende
responder a las
necesidades del \\ B7. La inclusión
educativa pretende
responder a las
necesidades del \\ B7. La inclusión
educativa pretende
responder a las
necesidades del \\ alumnado. \\ alumnado.}

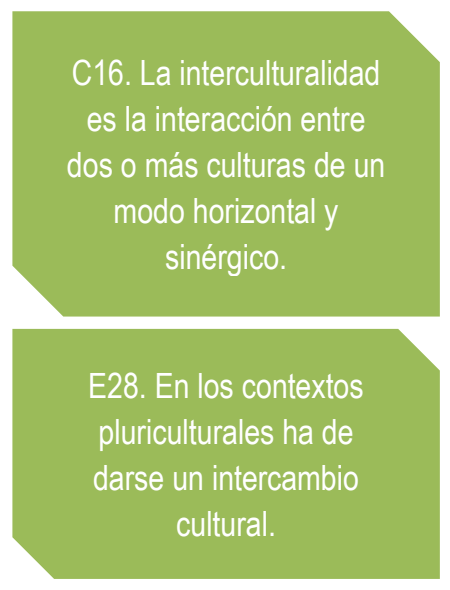

\section{C16. La interculturalidad}

es la interacción entre

dos o más culturas de un sinérgico. 
Fuente propia.

Se puede observar la coherencia en las correlaciones entre todos los ítems.

\section{4.-Análisis descriptivo.}

Dimensión A (Tendencias Científicas): por un lado, las personas participantes del Grado están de acuerdo ( $\bar{X}=4.5)$, por otro lado, los sujetos encuestados del Máster también están de acuerdo $(\bar{X}=4.2)$ en que la formación del profesorado en neuroeducación favorece la inclusión educativa.

Dimensión B (Inclusión Educativa): las personas encuestadas del Máster de Profesorado se muestran indiferente-de acuerdo $(\bar{X}=3.9)$, mientras que los sujetos encuestados del Grado se muestran indiferente $(\bar{X}=3.5)$ en que el profesorado dinamiza la inclusión en el aula.

Dimensión C (Pluriculturalidad): los sujetos encuestados tanto del Grado como del Máster se muestran en desacuerdo $(\bar{X}=2.2)$ en que la culturalidad es única e intransferible.

Dimensión D (Contextos Educativos): por una parte, los estudiantes encuestados del Máster opinan que están de acuerdo $(\bar{X}=4.5)$, mientras que las personas encuestadas del Grado se muestran indiferente-de acuerdo $(\bar{X}=3.9)$ en que los contextos educativos deben trabajar a favor de la inclusión.

Dimensión E (Contextos Pluriculturales): tanto las personas encuestadas del Máster como del Grado se muestran de acuerdo $(\bar{X}=4.4)$ en que los contextos pluriculturales deben favorecer el respeto y tolerancia a la diversidad cultural.

Dimensión $\mathrm{F}$ (Contextos Inclusivos): los sujetos encuestados del Grado se muestran de acuerdo $(\bar{X}=4.1)$, en cambio, las personas encuestadas del Máster se muestran indiferente $(\overline{\mathrm{X}}=3.5)$ en que en los contextos inclusivos se atiende la neurodiversidad del alumnado.

\section{4. -Discusión.}

Según los datos obtenidos, para resolver nuestro problema de investigación planteado, podemos afirmar que la neurociencia es adecuada para llevar a cabo una inclusión educativa en contextos educativos pluriculturales.

Por lo que los resultados obtenidos nos hacen pensar acerca de incluir la neurociencia en la educación para favorecer la inclusión en contextos pluriculturales para crear contextos inclusivos en la educación. Para ello, es importante la predisposición del profesorado, en su totalidad, para aprender sobre neurociencia e inclusión y practicarla en su enseñanza, para promover la pluriculturalidad y crear contextos educativos pluriculturales e inclusivos.

La investigación presentada se ha realizado para una población de Grado de Educación Primaria y de Máster de Profesorado con el objetivo de correlacionar los datos en niveles universitarios diferentes relacionados com la educación. En un futuro, sería conveniente realizar esta investigación a los profesionales educativos de los centros docentes para correlacionar los datos entre los estudiantes y los profesionales activos. 


\section{5.-Conclusión.}

La presente investigación toma como objetivo comparar las opiniones del alumnado de Grado de Educación Primaria y Máster de Profesorado en relación a la presencia de la neurociencia en el aula pluricultural para fomentar la inclusión. Para alcanzar esta finalidad se procede al diseño de una escala Likert a través de una tabla de operacionalización. Se realiza un análisis de fiabilidad dando un resultado bueno, en la escala de Grado, según el alpha de Conbach (.836) y un resultado aceptablebueno (.795) para la escala de Máster.

Posteriormente, tras comprobar con la prueba de Kolmogorov-Smirnov que la distribución de datos no es normal, en ambas escalas, por lo que se calcula la correlación Rho de Spearman. Resaltamos algunas correlaciones que consideramos de especial interés para la escala de Grado: las personas encuestadas que están de acuerdo en que la pluriculturalidad son diferentes culturas que existen en el mundo, también están de acuerdo que La transculturalidad es la interacción de costumbres de otras culturas para hacerlas propias (C14>C18). Para la escala de Máster podemos destacar: aquellos sujetos encuestados que están de acuerdo que en los contextos pluriculturales ha de darse un intercambio cultural, también están de acuerdo en que los contextos pluriculturales deben ser cooperativos y colaborativos (E28>E30).

El análisis descriptivo nos permite apreciar que los sujetos investigados, tanto del Grado como del Máster comparten las mismas ideas y pensamentos acerca de la neurociencia, la inclusión y la pluriculturalidad y la forma en la influye la neurociencia para crear una inclusión en contextos educativos pluriculturales.

\section{6.-Referencias.}

Ainscow, M. (2017). Haciendo que las escuelas sean inclusivas: lecciones a partir del análisis de la investigación internacional. Revista de Educación Inclusiva, 5(1), 39-49.

Arraya Pizarro, S.C., y Espinoza, L. (2020). Aportes desde las neurociencias para la comprensión de los procesos de aprendizaje en los contextos educativos. $\begin{array}{llll}\text { Propósitos } \quad \text { y } & \text { Representaciones, } & \text { 8(1), }\end{array}$ https://doi.org/10.20511/pyr2020.v8n1.312

De Barros, C., y Hernández, A. (2018). Educación inclusiva: bases neurocientíficas y tecnológicas en inclusión y transculturalidad. Jaén: Masquelibros.

Florian, L.., y Camedda, D. (2020) Enhancing teacher education for inclusion. European Journal of Teacher Education, 43(1), 4-8. https://doi.org/10.1080/02619768.2020.1707579

Gago, L., y Elgier, Á. (2018). Trazando puentes entre las neurociencias y la educación. Aportes, límites y caminos futuros en el campo educativo. Psicogente, 21(40), 476-494. https://doi.org/10.17081/psico.21.40.3087

Jane, T. J. (2019). La intraculturalidad en el espacio lusófono. Maremagnum: publicación galega sobre os trastornos do espectro autista. v.23, p.129-140.

Leiva, J. J. (2019). La Educación Intercultural como propuesta educativa democrática e inclusiva. En AIDIPE (Ed.), XIX Congreso Internacioal de Investigación 
Educativa: Investigación Comprometida para la Transformación Social. Madrid: AIDIPE.

Muntaner, J. J. (2019). La inclusión una apuesta social y educativa, en M. El Homrani, S.M. Arias, y I. Ávalos (Coords.), La inclusión: una apuesta educativa y social. Madrid: Wolters Kluwer.

Rahman, A.A.,y Woollard, J. (2019). Neurodiversity awareness: Is Malaysia there yet? Internacional Journal of Evaluation and Research in Education, 8(4), 676685. https://doi.org/10.11591/ijere.v8i4.20307

Vidal, M. I. (2019). Gamificación para la inclusión educativa. En REDINE (Ed.), Conference Proceedings CIVINEDU 2019: 3rd International Virtual Conference on Educational Research and Innovation: October 9-10, 2019 (p. 136). Madrid: Redine. 